\title{
Fault Attacks on RSA Signatures with Partially Unknown Messages
}

\author{
Jean-Sébastien Coron ${ }^{1}$, Antoine Joux ${ }^{2}$, Ilya Kizhvatov ${ }^{1}$, David Naccache ${ }^{3}$, \\ and Pascal Paillier ${ }^{4}$ \\ 1 Université du Luxembourg \\ 6, rue Richard Coudenhove-Kalergi, L-1359 Luxembourg, Luxembourg \\ \{jean-sebastien.coron, ilya.kizhvatov\}@uni.lu \\ 2 DGA and Université de Versailles \\ UVSQ PRISM 45 avenue des États-Unis, F-78035, Versailles CEDEX, France \\ antoine.joux@m $4 x$.org \\ 3 École normale supérieure \\ Département d'informatique, Groupe de Cryptographie \\ 45, rue d'Ulm, F-75230 Paris Cedex 05, France \\ david.naccache@ens.fr \\ 4 Gemalto, Cryptography \& Innovation \\ 6 , rue de la Verrerie, F-92447 Meudon sur Seine, France \\ pascal.paillier@gemalto.com
}

\begin{abstract}
Fault attacks exploit hardware malfunctions to recover secrets from embedded electronic devices. In the late 90's, Boneh, DeMillo and Lipton [6] introduced fault-based attacks on CRT-RSA. These attacks factor the signer's modulus when the message padding function is deterministic. However, the attack does not apply when the message is partially unknown, for example when it contains some randomness which is recovered only when verifying a correct signature.

In this paper we successfully extends RSA fault attacks to a large class of partially known message configurations. The new attacks rely on Coppersmith's algorithm for finding small roots of multivariate polynomial equations. We illustrate the approach by successfully attacking several randomized versions of the ISO/IEC 9796-2 encoding standard. Practical experiments show that a 2048-bit modulus can be factored in less than a minute given one faulty signature containing 160 random bits and an unknown 160-bit message digest.
\end{abstract}

Keywords: Fault attacks, digital signatures, RSA, Coppersmith's theorem, ISO/IEC 9796-2.

\section{Introduction}

\subsection{Background}

RSA 21] is undoubtedly the most common digital signature scheme used in embedded security tokens. To sign a message $m$ with RSA, the signer applies an

C. Clavier and K. Gaj (Eds.): CHES 2009, LNCS 5747, pp. 444 456, 2009.

(C) International Association for Cryptologic Research 2009 
encoding (padding) function $\mu$ to $m$, and then computes the signature $\sigma=$ $\mu(m)^{d} \bmod N$. To verify the signature, the receiver checks that $\sigma^{e}=\mu(m)$ $\bmod N$. As shown by Boneh, DeMillo and Lipton [6] and others (e.g. [18]), RSA implementations can be vulnerable to fault attacks, especially when the Chinese Remainder Theorem (CRT) is used; in this case the device computes $\sigma_{p}=\mu(m)^{d}$ $\bmod p$ and $\sigma_{q}=\mu(m)^{d} \bmod q$ and the signature $\sigma$ is computed from $\sigma_{p}$ and $\sigma_{q}$ by Chinese Remaindering.

Assuming that the attacker is able to induce a fault when $\sigma_{q}$ is computed while keeping the computation of $\sigma_{p}$ correct, one gets $\sigma_{p}=\mu(m)^{d} \bmod p$ and $\sigma_{q} \neq$ $\mu(m)^{d} \bmod q$ and the resulting (faulty) signature $\sigma$ satisfies

$$
\sigma^{e}=\mu(m) \bmod p, \quad \sigma^{e} \neq \mu(m) \quad \bmod q .
$$

Therefore, given one faulty $\sigma$, the attacker can factor $N$ by computing

$$
\operatorname{gcd}\left(\sigma^{e}-\mu(m) \bmod N, N\right)=p .
$$

Boneh et al.'s fault attack is easily extended to any deterministic RSA encoding, e.g. the Full Domain Hash (FDH) 5 ] encoding where $\sigma=H(m)^{d} \bmod N$ and $H:\{0,1\}^{*} \mapsto \mathbb{Z}_{N}$ is a hash function. The attack is also applicable to probabilistic signature schemes where the randomizer used to generate the signature is sent along with the signature, e.g. as in the Probabilistic Full Domain Hash (PFDH) encoding [11] where the signature is $\sigma \| r$ with $\sigma=H(m \| r)^{d} \bmod N$. In that case, given the faulty value of $\sigma$ and knowing $r$, the attacker can still factor $N$ by computing $\operatorname{gcd}\left(\sigma^{e}-H(m \| r) \bmod N, N\right)=p$.

\subsection{Partially-Known Messages: The Fault-Attacker's Deadlock}

However, if the message is not entirely given to the attacker the attack is thwarted, e.g. this may occur when the signature has the form $\sigma=(m \| r)^{d} \bmod$ $N$ where $r$ is a random nonce. Here the verifier can recover $r$ only after completing the verification process; however $r$ can only be recovered when verifying a correct signature. Given a faulty signature, the attacker cannot retrieve $r$ nor infer $(m \| r)$ which would be necessary to compute $\operatorname{gcd}\left(\sigma^{e}-(m \| r) \bmod N, N\right)=p$.

In other words, the attacker faces an apparent deadlock: recovering the $r$ used in the faulty signature $\sigma$ seems to require that $\sigma$ is a correctly verifiable signature. Yet, obviously, a correct signature does not factor $N$. These conflicting constraints cannot be conciliated unless $r$ is short enough to be guessed by exhaustive search. Inducing faults in many signatures does not help either since different $r$ values are used in successive signatures (even if $m$ remains invariant). As a result, randomized RSA encoding schemes are usually considered to be inherently immune against fault attacks.

\subsection{The New Result}

We overcome the deadlock by showing how to extract in some cases the unknown message part (UMP) involved in the generation of faulty RSA signatures. We develop several techniques that extend Boneh et al.'s attack to a large class of 
partially known message configurations. We nonetheless assume that certain conditions on the unknown parts of the encoded message are met; these conditions may depend on the encoding function itself and on the hash functions used. To illustrate our attacks, we have chosen to consider the ISO/IEC 9796-2 standard [16]. ISO/IEC 9796-2 is originally a deterministic encoding scheme often used in combination with message randomization (e.g. in EMV [13]). The encoded message has the form:

$$
\mu(m)=6 \mathrm{~A}_{16}\|m[1]\| H(m) \| \mathrm{BC}_{16}
$$

where $m=m[1] \| m[2]$ is split into two parts. We show that if the unknown part of $m[1]$ is not too large (e.g. less than 160 bits for a 2048-bit RSA modulus), then a single faulty signature allows to factor $N$ as in 6$]$. The new method is based on a result by Herrmann and May [12] for finding small roots of linear equations modulo an unknown factor $p$ of $N$; $[12$ is itself based on Coppersmith's technique [7] for finding small roots of polynomial equations using the LLL algorithm [19]. We also show how to extend our attack to multiple UMPs and to scenarii where more faulty signatures can be obtained from the device.

\subsection{The ISO/IEC 9796-2 Standard}

ISO/IEC 9796-2 is an encoding standard allowing partial or total message recovery [16 17. The encoding can be used with hash functions $H(m)$ of diverse digest sizes $k_{h}$. For the sake of simplicity we assume that $k_{h}$, the size of $m$ and the size of $N$ (denoted $k$ ) are all multiples of 8 . The ISO/IEC 9796-2 encoding of a message $m=m[1] \| m[2]$ is

$$
\mu(m)=6 \mathrm{~A}_{16}\|m[1]\| H(m) \| \mathrm{BC}_{16}
$$

where $m$ [1] consists of the $k-k_{h}-16$ leftmost bits of $m$ and $m[2]$ represents the remaining bits of $m$. Therefore the size of $\mu(m)$ is always $k-1$ bits. Note that the original version of the standard recommended $128 \leq k_{h} \leq 160$ for partial message recovery (see [16, §5, note 4). In [9], Coron, Naccache and Stern introduced an attack against ISO/IEC 9796-2; the authors estimated that attacking $k_{h}=128$ and $k_{h}=160$ would require respectively $2^{54}$ and $2^{61}$ operations. After Coron et al.'s publication, ISO/IEC 9796-2 was amended and the current official requirement (see [17]) is now $k_{h} \geq 160$. In a recent work Coron, Naccache, Tibouchi and Weinmann successfully attack the currently valid version of ISO/IEC 9796-2 10.

To illustrate our purpose, we consider a message $m=m[1] \| m[2]$ of the form

$$
m[1]=\alpha\|r\| \alpha^{\prime}, \quad m[2]=\text { DATA }
$$

where $r$ is a message part unknown to the adversary, $\alpha$ and $\alpha^{\prime}$ are strings known to the adversary and DATA is some known or unknown string 2 . The size of $r$ is

\footnotetext{
${ }^{1}$ In our attack, it does not matter how large the unknown part of $m[2]$ is.

2 The attack will work equally well in both cases.
} 
denoted $k_{r}$ and the size of $m[1]$ is $k-k_{h}-16$ as required in ISO/IEC 9796-2. The encoded message is then

$$
\mu(m)=6 \mathrm{~A}_{16}\|\alpha\| r\left\|\alpha^{\prime}\right\| H\left(\alpha\|r\| \alpha^{\prime} \| \text { DATA }\right) \| \mathrm{BC}_{16}
$$

Therefore the total number of unknown bits in $\mu(m)$ is $k_{r}+k_{h}$.

\section{Fault Attack on Partially-Known Message ISO/IEC 9796-2}

This section extends 6 to signatures of partially known messages encoded as described previously. We assume that after injecting a fault the opponent is in possession of a faulty signature $\sigma$ such that:

$$
\sigma^{e}=\mu(m) \quad \bmod p, \quad \sigma^{e} \neq \mu(m) \quad \bmod q .
$$

From (2) we can write

$$
\mu(m)=t+r \cdot 2^{n_{r}}+H(m) \cdot 2^{8}
$$

where $t$ is a known value. Note that both $r$ and $H(m)$ are unknown to the adversary. From (3) we obtain:

$$
\sigma^{e}=t+r \cdot 2^{n_{r}}+H(m) \cdot 2^{8} \bmod p .
$$

This shows that $(r, H(m))$ must be a solution of the equation

$$
a+b \cdot x+c \cdot y=0 \quad \bmod p
$$

where $a:=t-\sigma^{e} \bmod N, b:=2^{n_{r}}$ and $c:=2^{8}$ are known. Therefore we are left with solving equation (5) which is linear in the two variables $x, y$ and admits a small root $\left(x_{0}, y_{0}\right)=(r, H(m))$. However the equation holds modulo an unknown divisor $p$ of $N$ and not modulo $N$. Such equations were already exploited by Herrmann and May [12] to factor an RSA modulus $N=p q$ when some blocks of $p$ are known. Their method is based on Coppersmith's technique for finding small roots of polynomial equations [7. Coppersmith's technique uses LLL to obtain two polynomials $h_{1}(x, y)$ and $h_{2}(x, y)$ such that

$$
h_{1}\left(x_{0}, y_{0}\right)=h_{2}\left(x_{0}, y_{0}\right)=0
$$

holds over the integers. Then one computes the resultant between $h_{1}$ and $h_{2}$ to recover the common root $\left(x_{0}, y_{0}\right)$. To that end, we must assume that $h_{1}$ and $h_{2}$ are algebraically independent. This ad hoc assumption makes the method heuristic; nonetheless it turns out to work quite well in practice. Then, given the root $\left(x_{0}, y_{0}\right)$ one recovers the randomized encoded message $\mu(m)$ and factors $N$ by GCD. 
Theorem 1 (Herrmann-May [12]). Let $N$ be a sufficiently large composite integer with a divisor $p \geq N^{\beta}$. Let $f(x, y)=a+b \cdot x+c \cdot y \in \mathbb{Z}[x, y]$ be a bivariate linear polynomial. Assume that $f\left(x_{0}, y_{0}\right)=0 \bmod p$ for some $\left(x_{0}, y_{0}\right)$ such that $\left|x_{0}\right| \leq N^{\gamma}$ and $\left|y_{0}\right| \leq N^{\delta}$. Then for any $\varepsilon>0$, under the condition

$$
\gamma+\delta \leq 3 \beta-2+2(1-\beta)^{3 / 2}-\varepsilon
$$

one can find $h_{1}(x, y), h_{2}(x, y) \in \mathbb{Z}[x, y]$ such that $h_{1}\left(x_{0}, y_{0}\right)=h_{2}\left(x_{0}, y_{0}\right)=0$ over $\mathbb{Z}$, in time polynomial in $\log N$ and $\varepsilon^{-1}$.

We only sketch the proof and refer the reader to [12] and [8] for more details. Assume that $b=1$ in the polynomial $f$ (otherwise multiply $f$ by $b^{-1} \bmod N$ ) and consider the polynomial

$$
f(x, y)=a+x+c \cdot y
$$

We look for $\left(x_{0}, y_{0}\right)$ such that $f\left(x_{0}, y_{0}\right)=0 \bmod p$. The basic idea consists in generating a family $\mathcal{G}$ of polynomials admitting $\left(x_{0}, y_{0}\right)$ as a root modulo $p^{t}$ for some large enough integer $t$. Any linear combination of these polynomials will also be a polynomial admitting $\left(x_{0}, y_{0}\right)$ as a root modulo $p^{t}$. We will use LLL to find such polynomials with small coefficients. To do so, we view any polynomial $h(x, y)=\sum h_{i, j} x^{i} y^{j}$ as the vector of coefficients $\left(h_{i, j} X^{i} Y^{j}\right)_{i, j}$ and denote by $\|h(x X, y Y)\|$ this vector's Euclidean norm. Performing linear combinations on polynomials is equivalent to performing linear operations on their vectorial representation, so that applying LLL to the lattice spanned by the vectors in $\mathcal{G}$ will provide short vectors representing polynomials with root $\left(x_{0}, y_{0}\right) \bmod p^{t}$.

We now define the family $\mathcal{G}$ of polynomials as

$$
g_{k, i}(x, y):=y^{i} \cdot f^{k}(x, y) \cdot N^{\max (t-k, 0)}
$$

for $0 \leq k \leq m, 0 \leq i \leq m-k$ and integer parameters $t$ and $m$. For all values of indices $k, i$, it holds that $g_{k, i}\left(x_{0}, y_{0}\right)=0 \bmod p^{t}$. We first sort the polynomials $g_{k, i}$ by increasing $k$ values and then by increasing $i$ values. Denoting $X=N^{\gamma}$ and $Y=N^{\delta}$, we write the coefficients of the polynomial $g_{k, i}(x X, y Y)$ in the basis $x^{k^{\prime}} \cdot y^{i^{\prime}}$ for $0 \leq k^{\prime} \leq m$ and $0 \leq i^{\prime} \leq m-k^{\prime}$. Let $L$ be the corresponding lattice; $L$ 's dimension is

$$
\omega=\operatorname{dim}(L)=\frac{m^{2}+3 m+2}{2}=\frac{(m+1)(m+2)}{2}
$$

and we have

$$
\operatorname{det} L=X^{s_{x}} Y^{s_{y}} N^{s_{N}}
$$

where

$$
s_{x}=s_{y}=\sum_{k=0}^{m} \sum_{i=0}^{m-k} i=\frac{m(m+1)(m+2)}{6}
$$

and

$$
s_{N}=\sum_{i=0}^{t}(m+1-i) \cdot(t-i) \text {. }
$$


We now apply LLL to the lattice $L$ to find two polynomials $h_{1}(x, y)$ and $h_{2}(x, y)$ with small coefficients.

Theorem 2 (LLL [19]). Let $L$ be a lattice spanned by $\left(u_{1}, \ldots, u_{\omega}\right)$. Given the vectors $\left(u_{1}, \ldots, u_{\omega}\right)$, the LLL algorithm finds in polynomial time two linearly independent vectors $b_{1}, b_{2}$ such that

$$
\left\|b_{1}\right\|,\left\|b_{2}\right\| \leq 2^{\omega / 4}(\operatorname{det} L)^{1 /(\omega-1)} .
$$

Therefore using LLL we can get two polynomials $h_{1}(x, y)$ and $h_{2}(x, y)$ such that

$$
\left\|h_{1}(x X, y Y)\right\|,\left\|h_{2}(x X, y Y)\right\| \leq 2^{\omega / 4} \cdot(\operatorname{det} L)^{1 /(\omega-1)} .
$$

Using Howgrave-Graham's lemma (below), we can determine the required bound on the norms of $h_{1}$ and $h_{2}$ to ensure that $\left(x_{0}, y_{0}\right)$ is a root of both $h_{1}$ and $h_{2}$ over the integers:

Lemma 1 (Howgrave-Graham [14]). Assume that $h(x, y) \in \mathbb{Z}[x, y]$ is a sum of at most $\omega$ monomials and assume further that $h\left(x_{0}, y_{0}\right)=0 \bmod B$ where $\left|x_{0}\right| \leq X$ and $\left|y_{0}\right| \leq Y$ and $\|h(x X, y Y)\|<B / \sqrt{\omega}$. Then $h\left(x_{0}, y_{0}\right)=0$ holds over the integers.

Proof. We have

$$
\begin{aligned}
\left|h\left(x_{0}, y_{0}\right)\right| & =\left|\sum h_{i j} x_{0}^{i} y_{0}^{i}\right|=\left|\sum h_{i j} X^{i} Y^{j}\left(\frac{x_{0}}{X}\right)^{i}\left(\frac{y_{0}}{Y}\right)^{j}\right| \\
& \leq \sum\left|h_{i j} X^{i} Y^{j}\left(\frac{x_{0}}{X}\right)^{i}\left(\frac{y_{0}}{Y}\right)^{j}\right| \leq \sum\left|h_{i j} X^{i} Y^{j}\right| \\
& \leq \sqrt{\omega}\|h(x X, y Y)\|<B
\end{aligned}
$$

Since $h\left(x_{0}, y_{0}\right)=0 \bmod B$, this implies that $h\left(x_{0}, y_{0}\right)=0$ over the integers.

We apply Lemma 1 with $B:=p^{t}$. Using (7) this gives the condition:

$$
2^{\omega / 4} \cdot(\operatorname{det} L)^{1 /(\omega-1)} \leq \frac{N^{\beta t}}{\sqrt{\omega}} .
$$

[12 shows that by letting $t=\tau \cdot m$ with $\tau=1-\sqrt{1-\beta}$, we get the condition:

$$
\gamma+\delta \leq 3 \beta-2+2(1-\beta)^{3 / 2}-\frac{3 \beta(1+\sqrt{1-\beta})}{m}
$$

Therefore we obtain as in 12 the following condition for $m$ :

$$
m \geq \frac{3 \beta(1+\sqrt{1-\beta})}{\varepsilon} .
$$

Since LLL runs in time polynomial in the lattice's dimension and coefficients, the running time is polynomial in $\log N$ and $1 / \varepsilon$. 


\subsection{Discussion}

For a balanced RSA modulus $(\beta=1 / 2)$ we get the condition:

$$
\gamma+\delta \leq \frac{\sqrt{2}-1}{2} \cong 0.207
$$

This means that for a 1024-bit RSA modulus $N$, the total size of the unknowns $x_{0}$ and $y_{0}$ can be at most 212 bits. Applied to our context, this implies that for ISO/IEC 9796-2 with $k_{h}=160$, the size of the UMP $r$ can be as large as 52 bits. Section 3 reports practical experiments confirming this prediction. In [8] we provide a Python code for computing the bound on the size of the unknown values $\left(k_{r}+k_{h}\right)$ as a function of the modulus size.

\subsection{Extension to Several Unknown Bits Blocks}

Assume that the UMP used in ISO/IEC 9796-2 is split into $n$ different blocks, namely

$$
\mu(m)=6 \mathrm{~A}_{16}\left\|\alpha_{1}\right\| r_{1}\left\|\alpha_{2}\right\| r_{2}\|\cdots\| \alpha_{n}\left\|r_{n}\right\| \alpha_{n+1}\|H(m)\| \mathrm{BC}_{16}
$$

where the UMPs $r_{1}, \ldots, r_{n}$ are all part of the message $m$. The $\alpha_{i}$ blocks are known. In [8], we show how to recover $p$ from one faulty signature, using the extended result of Herrmann and May [12. It appears that if the total number of unknown bits plus the message digest is less than $15.3 \%$ of the size of $N$, then the UMPs can be fully recovered from the faulty signature and Boneh et al.'s attack will apply again. However the number of blocks cannot be too large because the attack's runtime increases exponentially with $n$.

\subsection{Extension to Two Faults Modulo Different Factors}

Assume that we can get two faulty signatures, one incorrect modulo $p$ and the other incorrect modulo $q$. This gives the two equations

$$
\begin{array}{ll}
a_{0}+b_{0} \cdot x_{0}+c_{0} \cdot y_{0}=0 & \bmod p \\
a_{1}+b_{1} \cdot x_{1}+c_{1} \cdot y_{1}=0 & \bmod q
\end{array}
$$

with small unknowns $x_{0}, y_{0}, x_{1}, y_{1}$. We show in 8 that by multiplying the two equations, we get a quadri-variate equation modulo $N$ which can be solved by linearization under the following bound:

$$
\gamma+\delta \leq \frac{1}{6} \cong 0.167
$$

This remains weaker than condition (9). However the attack is significantly faster because it works over a lattice of constant dimension 9. Moreover, the $16.7 \%$ bound is likely to lend itself to further improvements using Coppersmith's technique instead of plain linearization. 


\subsection{Extension to Several Faults Modulo the Same Factor}

To exploit single faults, we have shown how to use lattice-based techniques to recover $p$ given $N$ and a bivariate linear equation $f(x, y)$ admitting a small root $\left(x_{0}, y_{0}\right)$ modulo $p$. In this context, we have used Theorem 1 which is based on approximate GCD techniques from [15]. In the present section we would like to generalize this to use $\ell$ different polynomials of the same form, each having a small root modulo $p$. More precisely, let $\ell$ be a fixed parameter and assume that as the result of $\ell$ successive faults, we are given $\ell$ different polynomials

$$
f_{u}\left(x_{u}, y_{u}\right)=a_{u}+x_{u}+c_{u} y_{u}
$$

where each polynomial $f_{u}$ has a small root $\left(\xi_{u}, \nu_{u}\right)$ modulo $p$ with $\left|\xi_{u}\right| \leq X$ and $\left|\nu_{u}\right| \leq Y$. Note that, as in the basic case, we re-normalized each polynomial $f_{u}$ to ensure that the coefficient of $x_{u}$ in $f_{u}$ is equal to one. To avoid double subscripts, we hereafter use the Greek letters $\xi$ and $\nu$ to represent the root values. We would like to use a lattice approach to construct new multivariate polynomials in the variables $\left(x_{1}, \cdots, x_{\ell}, y_{1}, \cdots, y_{\ell}\right)$ with the root $R=\left(\xi_{1}, \cdots, \xi_{\ell}, \nu_{1}, \cdots, \nu_{\ell}\right)$. To that end we fix two parameters $m$ and $t$ and build a lattice on a family of polynomials $\mathcal{G}$ of degree at most $m$ with root $R$ modulo $B=p^{t}$. This family is composed of all polynomials of the form

$$
y_{1}^{i_{1}} y_{2}^{i_{2}} \cdots y_{\ell}^{i_{\ell}} f_{1}\left(x_{1}, y_{1}\right)^{j_{1}} f_{2}\left(x_{2}, y_{2}\right)^{j_{2}} \cdots f_{\ell}\left(x_{\ell}, y_{\ell}\right)^{j_{\ell}} N^{\max (t-j, 0)},
$$

where each $i_{u}, j_{u}$ is non-negative, $i=\sum_{u=1}^{\ell} i_{u}, j=\sum_{u=1}^{\ell} j_{u}$ and $0 \leq i+j \leq m$. Once again, let $L$ be the corresponding lattice. Its dimension $\omega$ is equal to the number of monomials of degree at most $m$ in $2 \ell$ unknowns, i.e.

$$
\omega=\left(\begin{array}{c}
m+2 \ell \\
2 \ell
\end{array}\right) .
$$

Since we have a common upper bound $X$ for all values $\left|\xi_{u}\right|$ and a common bound for all $\left|\nu_{u}\right|$ we can compute the lattice's determinant as

$$
\operatorname{det}(L)=X^{s_{x}} Y^{s_{y}} N^{s_{N}},
$$

where $s_{x}$ is the sum of the exponents of all unknowns $x_{u}$ in all occurring monomials, $s_{y}$ is the sum of the exponents of the $y_{u}$ and $s_{N}$ is the sum of the exponents of $N$ in all occurring polynomials. For obvious symmetry reasons, we have $s_{x}=s_{y}$ and noting that the number of polynomials of degree exactly $d$ in $\ell$ unknowns is $\left(\begin{array}{c}d+\ell-1 \\ \ell-1\end{array}\right)$ we find

$$
s_{x}=s_{y}=\sum_{d=0}^{m} d\left(\begin{array}{c}
d+\ell-1 \\
\ell-1
\end{array}\right)\left(\begin{array}{c}
m-d+\ell \\
\ell
\end{array}\right) .
$$

Likewise, summing on polynomials with a non-zero exponent $v$ for $N$, where the sum of the $j_{u}$ is $t-v$ we obtain

$$
s_{N}=\sum_{v=1}^{t} v\left(\begin{array}{c}
t-v+\ell-1 \\
\ell-1
\end{array}\right)\left(\begin{array}{c}
m-t+v+\ell \\
\ell
\end{array}\right) .
$$


As usual, assuming that $p=N^{\beta}$ we can find a polynomial with the correct root over the integers under the condition of formula (8).

Concrete Bounds: Using the notation of Theorem 1, we compute effective bounds on $\gamma+\delta=\log (X Y) / \log (N)$ from the logarithm of condition (8), dropping the terms $\sqrt{\omega}$ and $2^{\omega / 4}$ which become negligible as $N$ grows. For concrete values of $N$, bounds are slightly smaller. Dividing by $\log (N)$, we find

$$
s_{x} \cdot(\gamma+\delta)+s_{N} \leq \beta t \omega .
$$

Thus, given $k, t$ and $m$, we can achieve at best

$$
\gamma+\delta \leq \frac{\beta t \omega-s_{N}}{s_{x}}
$$

In [8], we provide the achievable values of $\gamma+\delta$ for $\beta=1 / 2$, for various parameters and for lattice dimensions $10 \leq \omega \leq 1001$.

Recovering the Root: With $2 \ell$ unknowns instead of two, applying usual heuristics and hoping that lattice reduction directly outputs $2 \ell$ algebraically independent polynomials with the prescribed root over the integers becomes a wishful hope. Luckily, a milder heuristic assumption suffices to make the attack work. The idea is to start with $K$ equations instead of $\ell$ and iterate the lattice reduction attack for several subsets of $\ell$ equations chosen amongst the $K$ available equations. Potentially, we can perform $\left(\begin{array}{c}K \\ \ell\end{array}\right)$ such lattice reductions. Clearly, since each equation involves a different subset of unknowns, they are all different. Note that this does not suffice to guarantee algebraic independence; in particular, if we generate more than $K$ equations they cannot be algebraically independent. However, we only need to ascertain that the root $R$ can be extracted from the available set of equations. This can be done, using Gröbner basis techniques, under the heuristic assumption that the set of equations spans a multivariate ideal of dimension zero i.e. that the number of solutions is finite.

Note that we need to choose reasonably small values of $\ell$ and $K$ to be able to use this approach in practice. Indeed, the lattice that we consider should not become too large and, in addition, it should be possible to solve the resulting system of equations using either resultants or Buchberger's algorithm which means that neither the degree nor the number of unknowns should increase too much.

Asymptotic Bounds: Despite the fact that we cannot hope to run the multipolynomial variant of our attack when parameters become too large, it is interesting to determine the theoretical limit of the achievable value of $\gamma+\delta$ as the number of faults $\ell$ increases. To that end, we assume as previously that $\beta=1 / 2$, let $t=\tau m$ and replace $\omega, s_{x}$ and $s_{N}$ by the following approximations:

$$
\omega \cong \frac{m^{2 \ell}}{(2 \ell) !}, \quad s_{x} \cong \sum_{d=0}^{m} \frac{d^{\ell}(m-d)^{\ell}}{(\ell-1) ! \ell !}, \quad s_{N} \cong \sum_{v=1}^{t} v \frac{(t-v)^{\ell-1}(m-t+v)^{\ell}}{(\ell-1) ! \ell !} .
$$


Table 1. Bound for the relative size $\gamma+\delta$ of the unknowns as a function of the number of faults $\ell$

\begin{tabular}{c||c|c|c|c|c|c|c|c|c|c}
$\ell$ & 1 & 2 & 3 & 4 & 5 & 6 & 7 & 8 & 9 & 10 \\
\hline$\gamma+\delta$ & 0.207 & 0.293 & 0.332 & 0.356 & 0.371 & 0.383 & 0.391 & 0.399 & 0.405 & 0.410
\end{tabular}

For small $\ell$ values we provide in Table 1 the corresponding bounds on $\gamma+\delta$. Although we do not provide further details here due to lack of space, one can show that the bound $\gamma+\delta$ tends to $1 / 2$ as the number of faults $\ell$ tends to infinity and that all $\gamma+\delta$ values are algebraic numbers.

\section{Simulation Results}

Assuming that fault injection can be performed on unprotected devices (see Section 4), we simulated the attack. In the experiment we generated faulty signatures (using the factors $p$ and $q$ ) and applied to them the attack's mathematical analysis developed in the previous sections to factor $N$. For our experimental results of physical fault injection see Section 4 .

\subsection{Single-Fault Attack Simulations}

We first consider a single-UMP, single-fault attack when $H=$ SHA-1 i.e. $k_{h}=160$. Using the SAGE library LLL implementation, computations were executed on a 2GHz Intel notebook.

Experimental results are summarized in Table 2, We see that for 1024-bit RSA, the randomizer size $k_{r}$ must be quite small and the attack is less efficient than exhaustive search 3 . However for larger moduli, the attack becomes more efficient. Typically, using a single fault and a 158-bit UMP, a 2048-bit RSA modulus was factored in less than a minute.

Table 2. Single fault, single UMP 160-bit digests $\left(k_{h}=160\right)$. LLL runtime for different parameter combinations.

\begin{tabular}{|c|r|r|r|r|r|}
\hline modulus size $k$ & UMP size $k_{r}$ & $m$ & $t$ & lattice dim. $\omega$ & runtime \\
\hline 1024 & 6 & 10 & 3 & 66 & 4 minutes \\
1024 & 13 & 13 & 4 & 105 & 51 minutes \\
1536 & 70 & 8 & 2 & 45 & 39 seconds \\
1536 & 90 & 10 & 3 & 66 & 9 minutes \\
2048 & 158 & 8 & 2 & 45 & 55 seconds \\
\hline
\end{tabular}

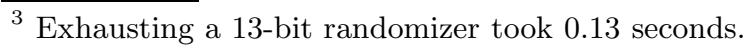




\subsection{Multiple-Fault Simulations}

To test the practicality of the approach presented in Section 2.4, we have set $(\ell, t, m)=(3,1,3)$ i.e. three faulty signatures. This leads to a lattice of dimension 84 and a bound $\gamma+\delta \leq 0.204$. Experiments were carried out with 1024, 1536 and 2048 bit RSA moduli. This implementation also relied on the SAGE library 20] running on a single PC. Quite surprisingly, we observed a very large number of polynomials with the expected root over the integers. The test was run for three random instances corresponding to the parameters in Table 3 .

Table 3. Three faults, single UMP, 160-bit digests $\left(k_{h}=160\right)$. LLL runtime for different parameter combinations.

\begin{tabular}{|c|r|r|}
\hline modulus size $k$ & UMP size $k_{r}$ & \multicolumn{1}{c|}{ runtime } \\
\hline 1024 & 40 & 49 seconds \\
1536 & 150 & 74 seconds \\
2048 & 250 & 111 seconds \\
\hline
\end{tabular}

Three faults turn-out to be more efficient than single-fault attacks (Table 3 vs. Table 21). In particular for a 1024-bit RSA modulus, the three-fault attack recovered a 40-bit UMP $r$ in 49 second\& 4 , whereas the single-fault attack only recovered a 13-bit UMP in 51 minutes.

\section{Physical Fault Injection Experiments}

We performed fault injection on an unprotected device to demonstrate the entire attack flow. We obtain a faulty signature from a general-purpose 8-bit microcontroller running an RSA implementation and factor $N$ using the mathematical attack of Section 2 .

Our target device is an Atmel ATmega128 [3, a very pupular RISC microcontroller $(\mu \mathrm{C})$ with an 8-bit AVR core. The $\mu \mathrm{C}$ was running an RSA-CRT implementation developed in $\mathrm{C}$ using the BigDigits multiple-precision arithmetic library [4. The $\mu \mathrm{C}$ was clocked at $7.3728 \mathrm{MHz}$ using a quartz crystal and powered from a $5 \mathrm{~V}$ source.

We induced faults using voltage spikes (cf. to [1] and 2] for such attacks on similar $\mu \mathrm{Cs}$ ). Namely, we caused brief power cut-offs (spikes) by grounding the chip's $\mathrm{V}_{\mathrm{cc}}$ input for short time periods. Spikes were produced by an FPGA-based board counting the $\mu \mathrm{C}$ 's clock transitions and generating the spike at a precise moment. The cut-off duration was variable with $10 \mathrm{~ns}$ granularity and the spike temporal position could be fine-tuned with the same granularity. The fault was heuristically positioned to obtain the stable fault injection in one of the RSA-CRT branches (computing $\sigma_{p}$ or $\sigma_{q}$ ). A 40ns spike is presented in Figure 1, Larger spike durations caused a $\mu$ C's reset.

\footnotetext{
${ }^{4}$ We estimate that exhaustive search on a 40-bit UMP would take roughly a year on the same single PC.
} 


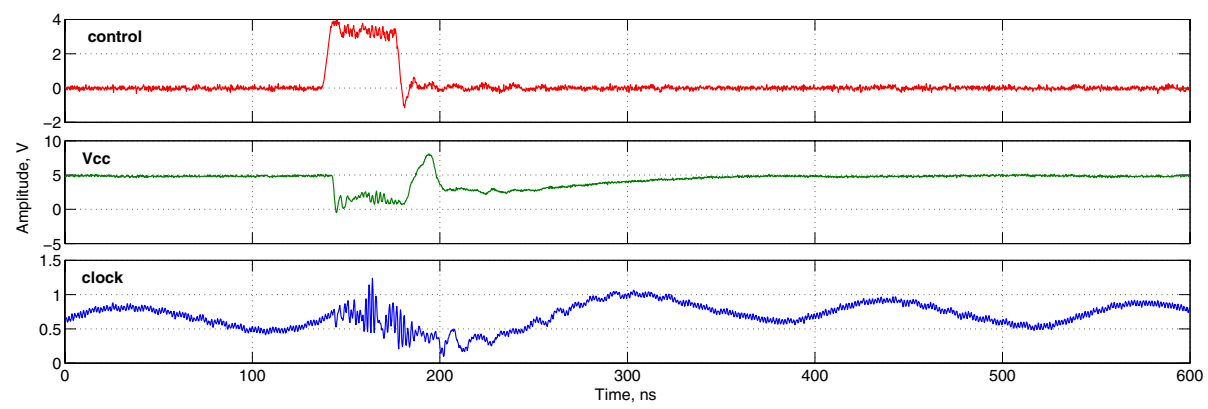

Fig. 1. Spike captured with a DSO: control signal from FPGA, power supply cut-off, and induced glitch in the clock signal

[8] provides more details on a 1536-bit RSA signature experiment conducted using our setup.

\section{Conclusion}

The paper introduced a new breed of partially-known message fault attacks against RSA signatures. These attacks allow to factor the modulus $N$ given a single faulty signature. Although the attack is heuristic, it works well in practice and paradoxically becomes more efficient as the modulus size increases. As several faulty signatures are given longer UMPs and longer digests become vulnerable.

\section{References}

1. Schmidt, J.-M., Herbst, C.: A practical fault attack on square and multiply. In: Proceedings of FDTC 2008, pp. 53-58. IEEE Computer Society Press, Los Alamitos (2008)

2. Kim, C.H., Quisquater, J.-J.: Fault attacks for CRT based RSA: New attacks, new results, and new countermeasures. In: Sauveron, D., Markantonakis, K., Bilas, A., Quisquater, J.-J. (eds.) WISTP 2007. LNCS, vol. 4462, pp. 215-228. Springer, Heidelberg (2007)

3. ATmega128 datasheet, http://www .atmel.com/dyn/resources/prod_documents/doc2467.pdf

4. BigDigits multiple-precision arithmetic source code, Version 2.2., http://www.di-mgt.com.au/bigdigits.html

5. Bellare, M., Rogaway, P.: The exact security of digital signatures - how to sign with RSA and rabin. In: Maurer, U.M. (ed.) EUROCRYPT 1996. LNCS, vol. 1070, pp. 399-416. Springer, Heidelberg (1996)

6. Boneh, D., DeMillo, R.A., Lipton, R.J.: On the importance of checking cryptographic protocols for faults. Journal of Cryptology 14(2), 101-119 (2001)

7. Coppersmith, D.: Small solutions to polynomial equations, and low exponent vulnerabilities. Journal of Cryptology 10(4), 233-260 (1997)

8. Coron, J.S., Joux, A., Kizhvatov, I., Naccache, D., Paillier, P.: Fault Attacks on Randomized RSA Signatures. Full version of this paper, http://eprint.iacr.org 
9. Coron, J.-S., Naccache, D., Stern, J.P.: On the security of RSA padding. In: Wiener, M. (ed.) CRYPTO 1999. LNCS, vol. 1666, pp. 1-18. Springer, Heidelberg (1999)

10. Coron, J.-S., Naccache, D., Tibouchi, M., Weinmann, R.P.: Practical cryptanalysis of ISO/IEC 9796-2 and EMV signatures. In: Halevi, S. (ed.) CRYPTO 2009. LNCS, vol. 5677, pp. 428-444. Springer, Heidelberg (2009), eprint.iacr.org/2009/203.pdf

11. Coron, J.-S.: Optimal security proofs for PSS and other signature schemes. In: Knudsen, L.R. (ed.) EUROCRYPT 2002. LNCS, vol. 2332, pp. 272-287. Springer, Heidelberg (2002)

12. Herrmann, M., May, A.: Solving linear equations modulo divisors: On factoring given any bits. In: Pieprzyk, J. (ed.) ASIACRYPT 2008. LNCS, vol. 5350, pp. 406-424. Springer, Heidelberg (2008)

13. EMV, Integrated circuit card specifications for payment systems, Book 2. Security and Key Management. Version 4.2 (June 2008), http://www.emvco.com

14. Howgrave-Graham, N.A.: Finding small roots of univariate modular equations revisited. In: Darnell, M.J. (ed.) Cryptography and Coding 1997. LNCS, vol. 1355, pp. 131-142. Springer, Heidelberg (1997)

15. Howgrave-Graham, N.A.: Approximate integer common divisors. In: CALC, pp. 51-66 (2001)

16. ISO/IEC 9796-2, Information technology - Security techniques - Digital signature scheme giving message recovery, Part 2: Mechanisms using a hash-function (1997)

17. ISO/IEC 9796-2:2002 Information technology - Security techniques - Digital signature schemes giving message recovery - Part 2: Integer factorization based mechanisms (2002)

18. Joye, M., Lenstra, A., Quisquater, J.-J.: Chinese remaindering cryptosystems in the presence of faults. Journal of Cryptology 21(1), 27-51 (1999)

19. Lenstra, A., Lenstra Jr., H., Lovász, L.: Factoring polynomials with rational coefficients. Mathematische Annalen 261, 513-534 (1982)

20. SAGE, Mathematical Library, http://www.sagemath.org

21. Rivest, R., Shamir, A., Adleman, L.: A method for obtaining digital signatures and public key cryptosystems. Communications of the ACM 21, 120-126 (1978) 